\title{
P 198 INTEGRATING PALLIATIVE CARE SERVICES IN LOW SOCIAL ECONOMIC STATUS
}

Asaph Kinyanjui. Kenya Hospices and Palliative Care Association, Nairobi, Kenya

10.1136/bmjspcare-2014-000654.239

To offer quality and sustainable palliative care services there is need to equip health care workers with appropriate knowledge, attitude and skills.

Waterloo Project The project was based on WHO Public Health Model.

The project was funded by The Diana Princess of Wales Memorial Fund and The True Colours Trust Fund. Other key stakeholders: Kenya Ministry of Health, Kenya Hospices and Palliative Care Associations, and African Palliative Care Association.

Back ground The overall purpose of the Waterloo project is to improve the quality of the lives of those affected by life threatening illnesses by ensuring freedom from pain and other distressing symptoms, providing psychosocial, spiritual and bereavement support through integration of palliative care in to the public health systems.

Method At the start of the project, a comprehensive data collection questionnaire was designed to collect baseline information on; hospitals with established palliative care services, type of services offered, staff trained in palliative care, availability of morphine and existence of a stand alone palliative care team. 11 level 5 hospitals were the focus of this project.

Successes Through KEHPCA and Ministry of Health the following have been achieved;

A) 9 hospitals in have fully integrated palliative services.

B) 220 healthcare workers have received 5 days training course.

C) More than 4oo doctors sensitized on palliative care especially pain management.

D) Inclusion of palliative care needs in some hospital budgets.

Challenges High turnover of hospital administrators in some hospitals affecting the integration of services.

Some staff trained in palliative care services deployed in non clinical areas.

Lack of essential palliative care medicines in the hospitals. 